\title{
Angiogenic factors FGF2 and PDGF-BB synergistically promote murine tumor neovascularization and metastasis
}

\author{
Lars Johan Nissen, ${ }^{1}$ Renhai Cao, ${ }^{1}$ Eva-Maria Hedlund, ${ }^{1}$ Zongwei Wang, ${ }^{1}$ \\ Xing Zhao, ${ }^{1}$ Daniel Wetterskog, ${ }^{2}$ Keiko Funa, ${ }^{2}$ Ebba Bråkenhielm, ${ }^{1}$ and Yihai Cao'1 \\ ${ }^{1}$ Department of Microbiology, Tumor and Cell Biology, Karolinska Institute, Stockholm, Sweden. \\ 2Institute of Biomedicine, Department of Medical Chemistry and Cell Biology, Göteborg University, Gothenburg, Sweden.
}

\begin{abstract}
Tumors produce multiple growth factors, but little is known about the interplay between various angiogenic factors in promoting tumor angiogenesis, growth, and metastasis. Here we show that 2 angiogenic factors frequently upregulated in tumors, PDGF-BB and FGF2, synergistically promote tumor angiogenesis and pulmonary metastasis. Simultaneous overexpression of PDGF-BB and FGF2 in murine fibrosarcomas led to the formation of high-density primitive vascular plexuses, which were poorly coated with pericytes and VSMCs. Surprisingly, overexpression of PDGF-BB alone in tumor cells resulted in dissociation of VSMCs from tumor vessels and decreased recruitment of pericytes. In the absence of FGF2, capillary ECs lacked response to PDGF-BB. However, FGF2 triggers PDGFR- $\alpha$ and $-\beta$ expression at the transcriptional level in ECs, which acquire hyperresponsiveness to PDGF-BB. Similarly, PDGF-BB-treated VSMCs become responsive to FGF2 stimulation via upregulation of FGF receptor 1 (FGFR1) promoter activity. These findings demonstrate that PDGF-BB and FGF2 reciprocally increase their EC and mural cell responses, leading to disorganized neovascularization and metastasis. Our data suggest that intervention of this non-VEGF reciprocal interaction loop for the tumor vasculature could be an important therapeutic target for the treatment of cancer and metastasis.
\end{abstract}

\section{Introduction}

Similar to growing healthy tissues, expansion of malignant tissues and tumor metastasis are dependent on neovascularization, which is accomplished by processes of angiogenesis, vasculogenesis, and vascular remodeling (1-13). The tumor vasculature usually consists of disorganized, leaky, premature, torturous, and hemorrhagic blood vessels that provide a structural basis for cancer cell invasion and spread $(1,3,9,14)$. These unusual features of tumor blood vessels represent the consequence of an imbalanced production of various angiogenic factors and the hypoxic environment within the tumor tissue. For example, VEGF is usually expressed at high levels in most tumors and has become an obviously therapeutic target for cancer therapy $(1,3,6,14)$. Indeed, most current antiangiogenic strategies for cancer therapy are based on blocking VEGF functions and anti-VEGF agents have successfully been used for the treatment of certain types of human cancers (15-17). However, tumors also produce multiple non-VEGF angiogenic factors, and anti-VEGF monotherapy could potentially encounter drug resistance, suggesting that tumors could use non-VEGF angiogenic factors to grow blood vessels $(18,19)$.

The tumor tissue consists of heterogeneous and genetically unstable malignant cells and a diversity of various other cell types including inflammatory cells, stromal cells, blood vessel ECs, lymphatic ECs, and VSMCs and/or pericytes, which are constantly exposed to hypoxic and stressful environments $(18,19)$. Both genetic instability of tumor cells and diversity of cell types determine expression of

Nonstandard abbreviations used: Ac-LDL, acetylated LDL; BCE, bovine capillary endothelial (cell); CAT, chloramphenicol acetyltransferase; FGFR, FGF receptor; NG2, neuro/glial cell 2 chondroitin sulfate proteoglycan.

Conflict of interest: The authors have declared that no conflict of interest exists. Citation for this article: J. Clin. Invest. 117:2766-2777 (2007). doi:10.1172/JCI32479. multiple angiogenic factors in the tumor tissue (20). Both PDGF-BB and FGF2 are frequently expressed at high levels in various tumor tissues $(21,22)$. While PDGF-BB displays potent biological activity on PDGFR-expressing VSMCs, it usually lacks biological effects on ECs that do not express detectable levels of PDGFRs $(21,23,24)$. Thus PDGF-BB is considered as a mitogenic and chemotactic factor for VSMCs/pericytes, but not for ECs. Indeed, deletion of PDGF-B or its prominent receptor, PDGFR- $\beta$ in mice leads to embryonic lethality, manifesting leaky and hemorrhagic phenotypes due to lack of pericytes and/or VSMCs in blood vessels $(23,24)$. In contrast to PDGF-BB, FGF2 is a potent angiogenic factor directly stimulating EC proliferation, although it also acts on VSMCs in vitro (25). However, delivery of FGF2 in vivo mainly induces angiogenesis without significantly increasing recruitment of VSMCs (26). Although the roles of individual angiogenic factors in promoting tumor angiogenesis are relatively well studied, little is known about the interplay between various angiogenic factors and their combined effects in tumor neovascularization, growth, and metastasis. The tumor vasculature is constantly exposed to multiple growth factors, and the complex interactions between various factors determine the ultimate outcome of tumor vessel growth, which might involve activation of MAPK and other signaling components in ECs and other vascular cells (27).

In this study, we provide compelling evidence that FGF2 acts as a sensitizer for ECs to respond to PDGF-BB, which feeds back to VSMCs to enhance their responses to FGF2 stimulation. The underlying mechanisms of this reciprocal interaction involve upregulation of PDGFR expression in ECs by FGF2 and of FGFR1 expression in VSMCs by PDGF-BB. The biological consequence of such a reciprocal interaction in tumors is manifested by hyperneovascularization and high degree of disorganized primitive tumor vasculatures, which are poorly coated with pericytes and VSMCs. These altera- 
A
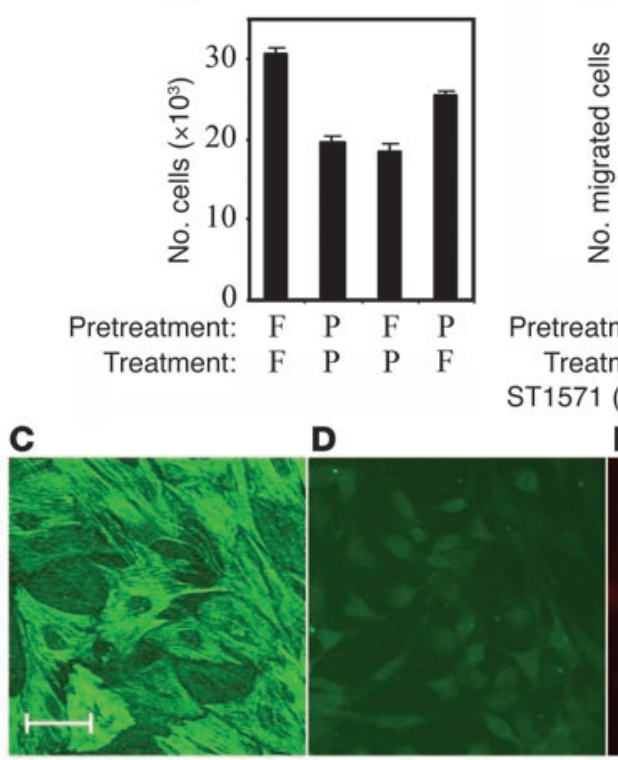

G

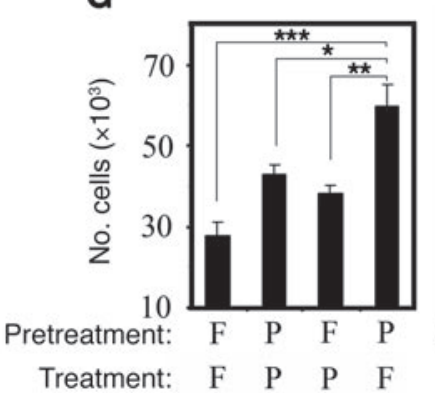

B

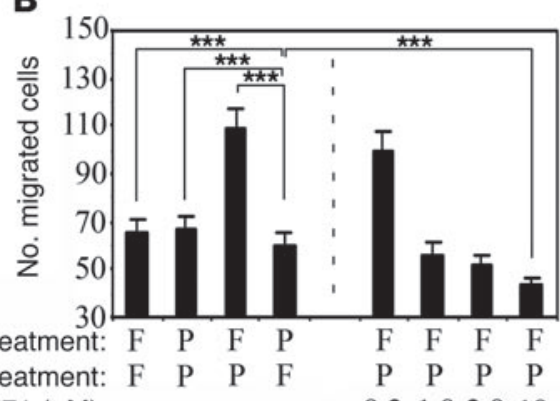

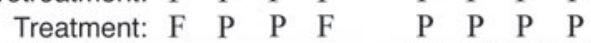

E
$\mathbf{F}$

\section{Figure 1}

Reciprocal regulation of proliferation and migration of ECs and VSMCs. (A) BCE cell proliferation. BCE cells were pretreated with FGF2 $(F)$ or PDGF-BB (P), followed by stimulation with either FGF2 or PDGF-BB. Cell proliferation was measured 72 hours after treatment by counting cell numbers. (B) Migration of FGF2or PDGF-BB-pretreated BCE cells were assayed in Boyden chambers for 4 hours in the presence and absence of STI571. Migrating cells were counted under a light microscope. (C-F) Rat VSMCs (C) and BCE cells (E) were immunostained with an anti- $\alpha$-SMA antibody. Uptake of 1,1'-dioctadecyl-3,3,3',3'-tetramethylindocarbocynaine perchlorate-labeled Ac-LDL by VSMCs (D) and BCE cells (F) was analyzed. ( $\mathbf{G}$ and $\mathbf{H}$ ) VSMC proliferation (G) and migration (H) stimulated by FGF2 and/or PDGF-BB. The data represent means of average determinants \pm SEM. ${ }^{*} P<0.05 ;{ }^{* \star} P<0.01 ;{ }^{* \star \star} P<0.001$. Scale bar: $50 \mu \mathrm{m}$. tions of tumor blood vessels lead to an accelerated tumor growth rate and metastasis. Thus our results provide what we believe to be new mechanistic insights on the cooperative role of various angiogenic factors in promoting tumor growth and metastasis.

\section{Results}

Prerequisite role of FGF2 in facilitating PDGF-BB-induced capillary EC migration. To study EC responses to FGF2 and PDGF-BB stimulation, bovine capillary endothelial (BCE) cells were used for in vitro studies. Previous studies have demonstrated that these capillary ECs were highly relevant for in vivo angiogenesis and were sensitive to various angiogenic stimuli and inhibitors $(28,29)$. As expected, FGF2 at a low concentration $(10 \mathrm{ng} / \mathrm{ml})$ significantly stimulated BCE cell proliferation (Figure 1A). In contrast, PDGF-BB exhibited a minor proliferative effect on these cells at a high concentration (100 $\mathrm{ng} / \mathrm{ml})$, but pretreatment of BCE with FGF2 did not significantly potentiate the effect of PDGF-BB on cell proliferation. Similarly, pretreatment of BCE cells with PDGF-BB did not increase BCE responses to FGF2 stimulation. Interestingly, FGF2 did not significantly induce BCE cell migration, suggesting that FGF2 acts as a mitogenic factor but not a chemotactic factor for ECs (Figure 1B). These results are consistent with previous findings that FGF2 is mainly a proliferative growth factor for capillary ECs (30). In the absence of FGF2, PDGF-BB did not significantly induce BCE cell motility. However, PDGF-BB remarkably stimulated the migration of BCE cells preexposed to FGF2, and this migratory effect could be inhibited by an anti-PDGFR agent, STI571, in a dose-dependent manner (Figure 1B). These results show that pretreatment of capillary ECs with FGF2 is a prerequisite for PDGF-BB-induced EC migration.

FGF2 and PDGF-BB synergistically induced angiogenesis. Reciprocal interactions between FGF2 and PDGF-BB on ECs and VSMCs suggested that these 2 factors cooperatively regulated vessel growth in vivo. To study this possibility, FGF2 and PDGF-BB were coimplanted into the mouse cornea. Indeed, FGF2 plus PDGF-BB synergistically induced corneal angiogenesis (Figure 2). Confocal analysis of whole-mount CD31-stained corneal tissues showed that at day 5 after implantation, FGF2 plus PDGF-BB-induced vessels consisted of a relatively disorganized vasculature as compared with single factor-induced vessels (Figure 2, E-G and I-K). We should emphasize that the disorganized vasculatures could only be detected at the early phase of the vessel formation, and they were remodeled into a well-defined tree-like vascular network after long-term exposure to FGF2 and PDGF-BB (31).

Transcriptional regulation of PDGFRs in ECs by FGF2. The FGF2-triggered onset of endothelial and angiogenic responses to PDGF-BB suggested that FGF2 might modulate PDGFR expression and signaling pathways in ECs. To study this possibility, promoters of mouse PDGFR- $\alpha$ and PDGFR- $\beta$ genes were cloned and fused with the luciferase reporter gene so that the PDGFR promoters would control the expression of luciferase. Transfection of these constructs into BCE cells resulted in dramatic activation of the 

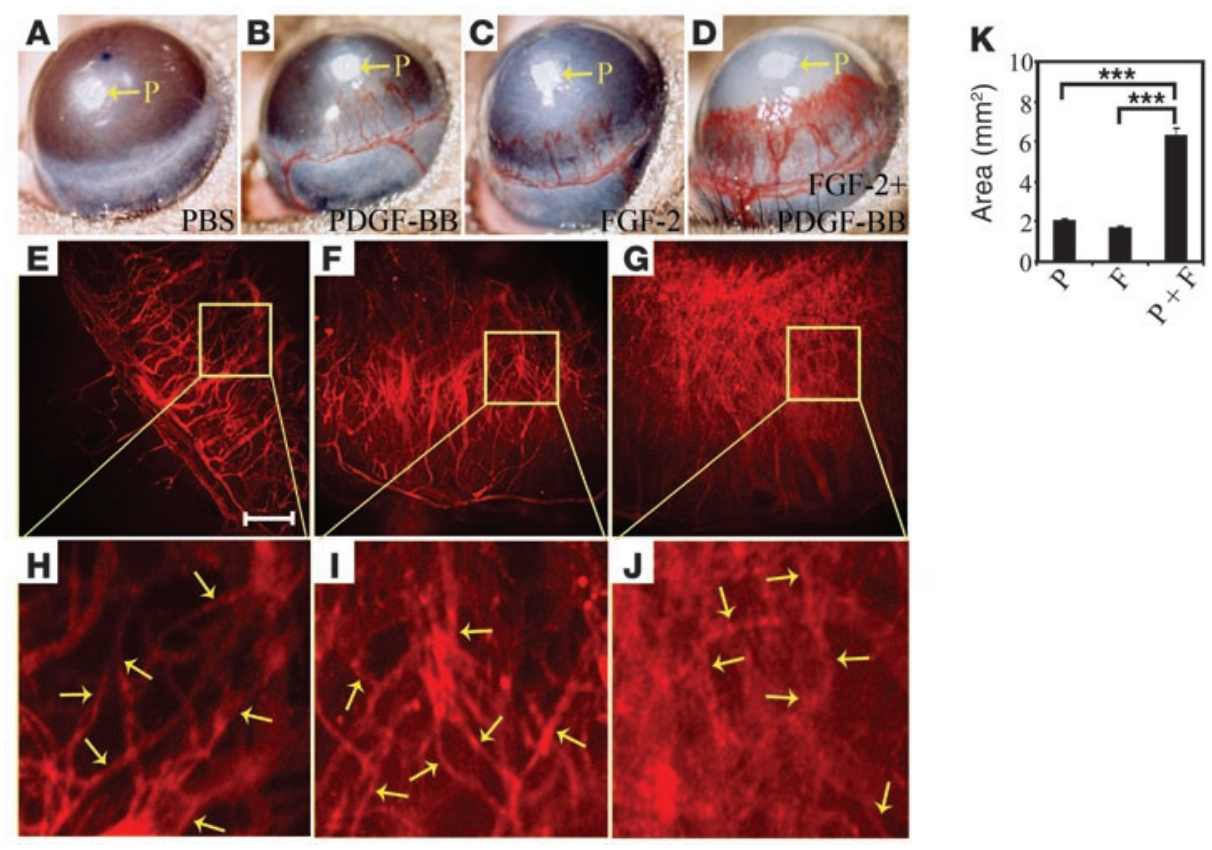

\section{Figure 2}

Angiogenic synergism. PBS (A), PDGF-BB (B and $\mathbf{E})$, FGF2 (C and F), or PDGF-BB plus FGF2 ( $\mathbf{D}$ and $\mathbf{G}$ ) together with a slow-release polymer was implanted into the mouse cornea, and corneal neovascularization was photographed on day 5 after implantation. (E-K) Growth factor-implanted corneas were stained with an anti-CD31 antibody, and corneal neovascularization was quantified by measuring vessel areas from 10-12 animals. Arrows point to CD31-positive microvessels. The data represent means of average determinants \pm SEM. ${ }^{* \star *} P<0.001$. Scale bar: $100 \mu \mathrm{m}(\mathbf{A}-\mathbf{G})$; $25 \mu \mathrm{m}(\mathbf{H}-\mathbf{J})$.

promoter activity of both PDGFR- $\alpha$ and PDGFR- $\beta$ in FGF2-treated cells but not in PDGF-BB-treated cells (Figure 3, A and B). To define the critical regions of the PDGFR promoter responsible for FGF2 activation in ECs, serial deletions or mutations were introduced into different regions of the PDGFR- $\beta$ promoter (Figure 3, $\mathrm{D}-\mathrm{G})$. Deletion or mutation of the $5^{\prime}$-end region, the GC-rich box, or the CAAT box resulted in loss of the promoter activity in FGF2treated BCE cells (Figure 3, D-G). Upregulation of PDGFR- $\alpha$ and PDGFR- $\beta$ by FGF2 in BCE cells was further validated by RT-PCR analysis (Figure 4A). These data demonstrate that the 5 '-end region, the GC-rich box, and the CAAT box are all crucial for FGF2-triggered upregulation of PDGFR expression.

To validate these in vitro findings, various angiogenic factorinduced corneal blood vessels were detected in situ for mRNA expression of PDGFRs. Consistent with the in vitro EC data, extremely high levels of both PDGFR- $\alpha$ and PDGFR- $\beta$ were found in the FGF2-induced blood vessels (Figure 4, C-J). ECs in the FGF2-induced new blood vessels expressed high levels of both PDGFR- $\alpha$ and PDGFR- $\beta$. In contrast, PDGF-BB-induced vessels only expressed a moderate level of PDGFRs. These in vivo findings provide further evidence that FGF2 at the transcriptional level triggers PDGF-BB-induced EC and blood vessel growth.

Elevated protein levels of PDGFR- $\beta$ in FGF2-treated ECs. To investigate if the elevated mRNA levels of PDGFRs in FGF2-treated BCE cells also led to increased protein expression, cell lysates treated with different concentrations of FGF2 were immunoblotted with an anti-PDGFR- $\beta$-specific antibody. At the concentrations of 5 and $10 \mathrm{ng} / \mathrm{ml}$, FGF2 remarkably increased PDGFR- $\beta$ expression in BCE cells (Figure 4B). In addition to elevation of total receptor molecules, the phosphorylated PDGFR- $\beta$ molecules were also proportionally increased, suggesting that high levels of PDGFR- $\beta$ led to activation of these receptors.

Activation of intracellular signaling pathways. Treatment of BCE cells with FGF2 led to an elevated level of phosphorylated Erk ( $\mathrm{p}$-Erk), which could be further increased following PDGF-BB treatment. High levels of p-Erk persisted for more than 6 hours before returning to the basal level (Figure 4K). A modest and transient effect of PDGF-BB alone on elevation of p-Erk was also detected (data not shown). Interestingly, after 60 minutes of exposure to PDGF-BB, the FGF2-pretreated cells began to show an elevated level of phospholipase C- $\gamma$ (PLC- $\gamma$ ), and high levels of PLC- $\gamma$ persisted for the entire time course of experiments. Levels of phosphorylated Src and Akt were not altered by FGF2 pretreatment. These findings validate that PDGF-BB directly induces EC activity in FGF2-pretreated cells.

PDGF-BB potentiated FGF2-induced VSMC proliferation. To study the role of PDGF-BB in modulating biological effects of FGF2 on VSMCs, we isolated rat aorta VSMCs, which expressed $\alpha$-SMA but lacked an ability of internalizing acetylated LDL (Ac-LDL) as BCE cells (Figure 1, C-F). As expected, both FGF2 and PDGF-BB significantly stimulated VSMC proliferation and migration (Figure 1, $\mathrm{G}$ and $\mathrm{H}$ ). Pretreatment of FGF2 did not alter PDGF-BB-induced VSMC proliferation and migration responses as compared with the FGF2-treated group. Remarkably, pretreatment of VSMCs with PDGF-BB significantly potentiated FGF2-induced cell proliferation but not cell migration (Figure $1, \mathrm{G}$ and $\mathrm{H}$ ). These findings demonstrate that PDGF-BB modulates FGF2-induced cell proliferation activity on VSMCs.

PDGF-BB induced FGFR1 promoter activity in VSMCs. To study if FGF2 and PDGF-BB reciprocally communicate with each other at the receptor signaling level, we studied the regulation of FGFR expression by PDGF-BB in both ECs and VSMCs. The promoter region of FGFR1 was fused with the chloramphenicol acetyltransferase (CAT) reporter gene, and the fusion construct was used to transfect both ECs and VSMCs. Interestingly, PDGF-BB induced about a 3 -fold increase of reporter gene activity in VSMCs (Figure 3C). The PDGF-BB-induced FGFR1 activity seemed to be restricted to VSMCs, and stimulation of FGFR1/ CAT-transfected BCE cells with PDGF-BB did not result in any increase of promoter activity (data not shown). In contrast to FGFR1, PDGF-BB did not induce promoter activity of FGFR3 and FGFR4 when assayed by promoter luciferase activity (data 


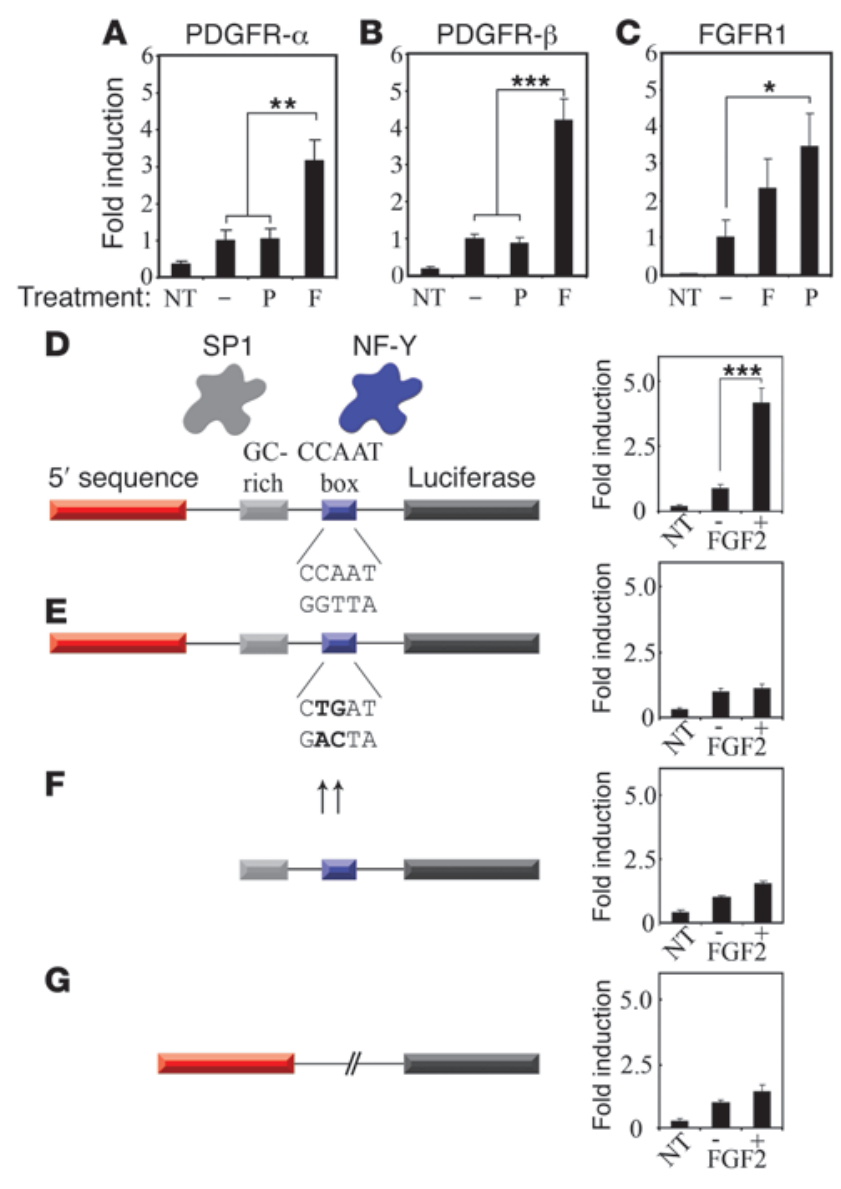

not shown). Thus our data indicate that PDGF-BB may upregulate FGFR1 expression in VSMCs but not in ECs.

FGF2 and PDGF-BB synergistically stimulated tumor neovascularization. To study the reciprocally interactive role of FGF2 and PDGF-BB in promoting tumor angiogenesis, secreted forms of FGF2 and PDGF-BB were overexpressed in a murine fibrosarcoma. As expected, implantation of PDGF-BB tumors in syngeneic mice resulted in an accelerated tumor growth rate as compared with nontransfected tumors (Figure 5A). Implantation of both PDGF-BB and FGF2 tumors in mice led to a further increase of the tumor growth rate. Immunohistochemical analysis of tumor tissues revealed an increased vascular density in FGF2- and PDGF-BB-overexpressing tumors (Figure 5, B and C). Strikingly, in the FGF2- and PDGF-BB-coexpressing tumors, not only was the vascular density remarkably increased, but the tumor vascular structure also underwent remarkable changes (Figure 5B). The FGF2/PDGF-BBinduced tumor blood vessels appeared to be primitive vascular plexuses, which exhibited disorganized large pseudo-vessels. Intriguingly, high numbers of capillary sprouts or tip cells existed in these primitive vascular plexuses, suggesting coordinated effects of these 2 factors in promoting tumor vessel growth. These findings demonstrate that FGF2 and PDGF-BB together not only synergistically stimulate tumor neovascularization but also change the architecture of the tumor vasculature.

Uncoupling of tumor microvessel growth and VSMC coating. PDGF-BB has previously been reported to be a potent mitogen for VSMCs (21). However, overexpression of PDGF-BB alone in tumors did not significantly increase the number of $\alpha$-SMA-positive structures

\section{Figure 3}

Regulation of PDGFR and FGFR promoter activity. PDGFR- $\alpha(\mathbf{A})$ and PDGFR- $\beta$ (B) promoters were fused with luciferase gene as a reporter system to detect promoter activity in ECs. After transfection, BCE cells were incubated with PDGF-BB or FGF2 for 40 hours, and luciferase activity was determined. NT, nontransfected. Dash indicates PBS treated. (C) Rat VSMCs were transfected with FGFR1-CAT construct and FGFR1 promoter activity was determined by measuring the CAT activity. (D-G) The intact (D) and various mutated (E-G) PDGFR- $\beta$ promoters were fused with luciferase reporter gene, and promoter activity was measured in growth factor-stimulated BCE cells. A $\beta$-galactosidase construct was used as a control to standardize the transfection system in all experiments. The data represent means of average determinants \pm SEM. ${ }^{\star} P<0.05$, ${ }^{\star *} P<0.01$, and ${ }^{* *} P<0.001$.

(Figure 6, A and B). Surprisingly, PDGF-BB promoted disassociation of VSMCs from tumor microvessels, which exhibited random distribution of VSMCs throughout the tumor tissue (Figure 6A). Although FGF2 and PDGF-BB together slightly increased the percentage of the association between CD31-positive structures and VSMCs, the total number of tumor vessels coated with VSMCs was significantly lower than that of controls (Figure 6A). In contrast, a nearly completely overlapping pattern of CD31- and $\alpha$-SMA-positive structures was seen in vector plus FGF2 tumors (Figure 6, A and C). Similarly, virtually all microvessels of vector and FGF2 tumors were coated with VSMCs. These data demonstrate surprising findings that expression of PDGF-BB in tumor cells results in disassociation of VSMCs from the tumor vasculature.

Inhibition of pericyte recruitment by tumor-produced PDGF-BB. We then analyzed pericyte distribution within the tumor tissue. In the vector-transfected tumors, a significant number of neuro/glial cell 2 chondroitin sulfate proteoglycan-positive (NG2-positive) pericytes was found in the tumor tissue, and virtually all of them remained in association with tumor microvessels (Figure 7, A-C). FGF2 significantly increased both the total number of pericytes in the tumor tissue and their recruitment onto tumor microvessels. In contrast, very few NG2-positive pericytes were present in PDGF-BB tumors, but almost all of them remained in association with tumor vessels (Figure 7, A-C). Similarly, only very sparsely distributed pericytes were found in the PDGF-BB and FGF2-coexpressing tumor tissue, and they mainly remained in association with relatively large tumor vessels. Almost all microvessels in PDGF-BB and PDGF-BB plus FGF2 tumors lacked pericyte coating. These unexpected findings demonstrate that PDGF-BB inhibits pericyte recruitment in the tumor environment.

Reciprocal vascular interactions between FGF2 and PDGF-BB promoted metastasis. To study the functional consequence of FGF2 plus PDGF-BB on tumor vasculature, we performed experiments of spontaneous metastasis in mice in which subcutaneous primary tumors were surgically removed at the size of $1.5 \mathrm{~cm}^{3}$. FGF2 tumor cells were labeled with luciferase, and PDGF-BB tumor cells were labeled with GFP for monitoring tumor spreads. Approximately 4 weeks after removal of primary tumors, about $30 \%$ of animals in the FGF2 plus PDGF-BB group developed pulmonary luciferase-positive metastatic nodules as detected by bioluminescence analysis (Figure 8, B and E). In contrast, FGF2 tumor-bearing mice or vector plus FGF2 tumor-bearing mice did not show any signs of lung metastasis (Figure 8A). These findings demonstrate that PDGF-BB could facilitate metastasis of FGF2 tumor cells despite the fact that PDGF-BB displayed no effects on tumor cells in vitro. Necropsy analysis of tumor-bearing 

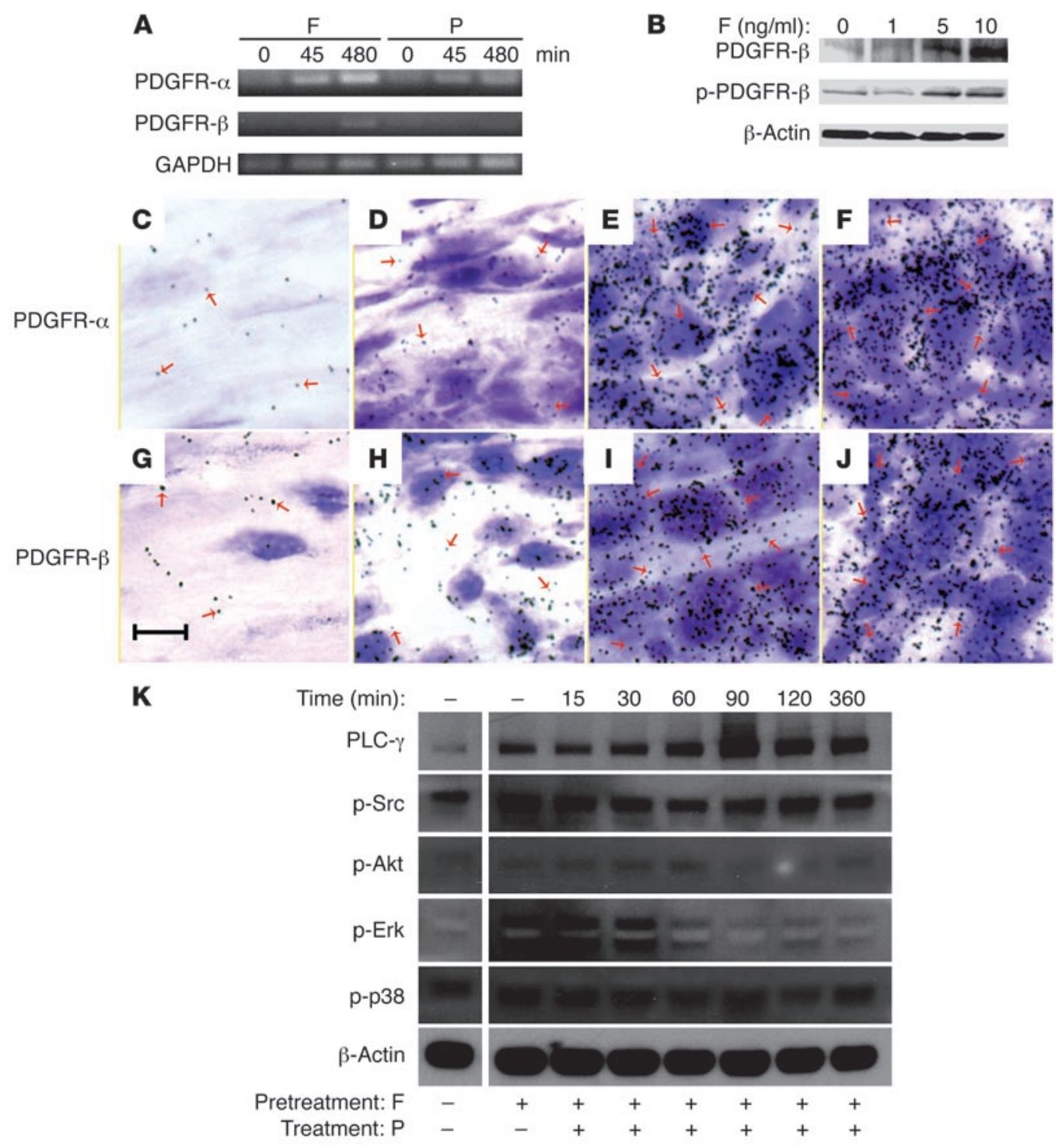

Figure 4

Regulation of mRNA and protein expression of PDGFRs and signaling pathways. (A) RT-PCR was used to quantitatively measure PDGFR- $\alpha$ and PDGFR- $\beta$ expression levels in FGF2- or PDGF-BB-treated BCE cells for the indicated time points. GAPDH was used as a standard control. (B) BCE cell lysates treated with different concentrations of FGF2 were immunoblotted with an anti-PDGFR- $\beta$ antibody and an anti-p-PDGFR- $\beta$ antibody. An anti- $\beta$-actin antibody was used as a standard control for loading levels. (C-J) In situ hybridization. PBS- (C and G), PDGF-BB- (D and H), FGF2- (E and I), or PDGF-BB/FGF2-implanted (F and $\mathbf{J})$ corneas were hybridized in situ with probes for PDGFR- $\alpha$ (C-F) and PDGFR- $\beta$ $(\mathbf{G}-\mathbf{J})$ at day 5 after growth factor implantation. Arrows point to positive signals. (K) Analysis of signaling components. FGF2-pretreated BCE cells were treated with PDGF-BB for indicated time points, and cell lysates were immunoblotted with different antibodies. $\beta$-Actin was immunoblotted as a control for loading levels. Scale bar: $10 \mu \mathrm{m}$.

mice revealed that about $30 \%$ of FGF2 plus PDGF-BB tumor-bearing mice had visible surface lung metastases (Figure 8, D and F). In contrast, none of the vector plus FGF2 tumor-bearing mice developed visible pulmonary lung metastasis (Figure 8, C and F). Histological analysis of lung tissues confirmed that FGF2 plus PDGF-BB stimulated lung metastases (Figure $8, \mathrm{H}$ and I). Because vector plus PDGF-BB tumor cells also expressed GFP, we detected GFP-positive tumor cells in the lung tissue. Interestingly, about $44 \%$ of mice showed GFP-positive pulmonary micrometastases, and none of the vector plus PDGF-BB tumor-bearing mice had GFP-positive cells in their lungs (Figure 8, G, J, and K). These findings show that recipro- cal interplay between FGF2 and PDGF-BB in the tumor environment promotes pulmonary tumor metastasis.

\section{Discussion}

Both genetic and epigenetic factors contribute to the switch of an angiogenic phenotype in tumors, which produce multiple angiogenic factors. Although angiogenic activity of individual factors is relatively well studied, little is known about the interplay between various tumor-produced angiogenic factors and their cooperative efforts in promoting tumor neovascularization. Here we report on the impact of the interplay between FGF2 and PDGF-BB on vas- 
A

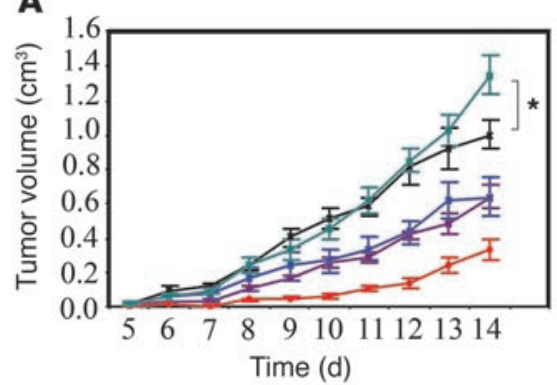

B

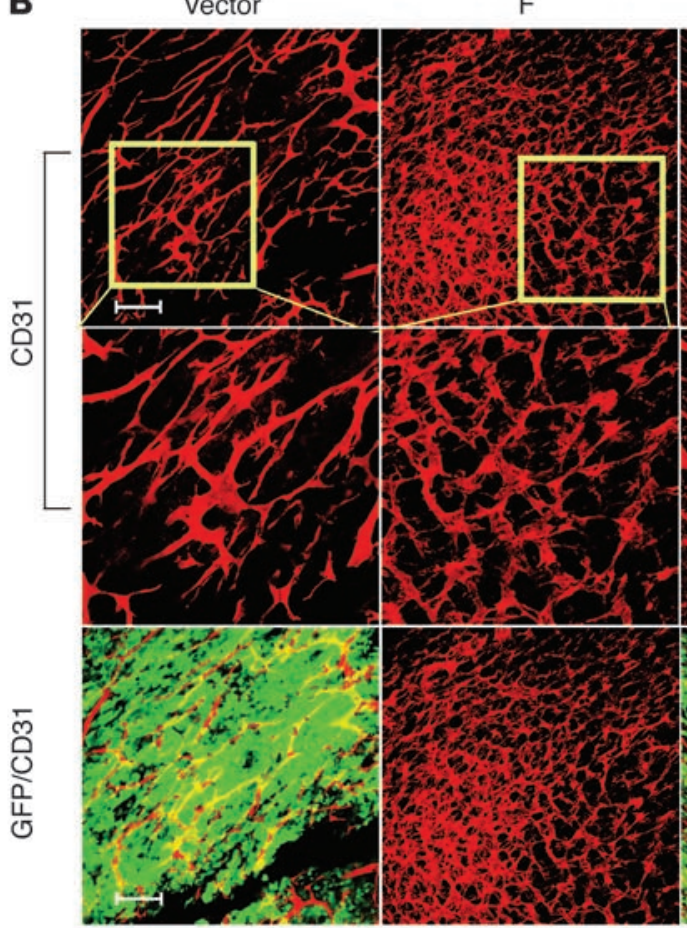

\section{C}

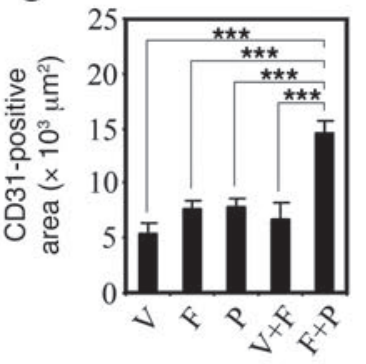

Vector $+\mathrm{F}$
$P+F$

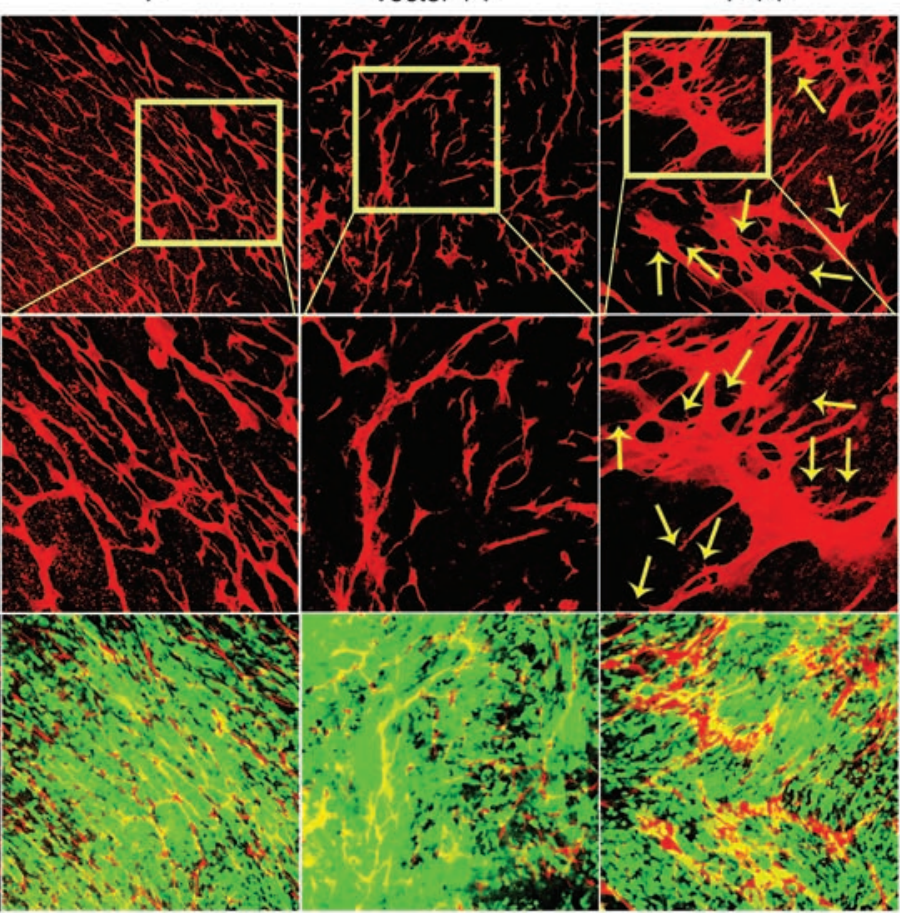

\section{Figure 5}

Tumor growth rates and vasculature. (A) Growth factor- or vector-transfected tumor cells were subcutaneously implanted in SCID mice, and tumor growth was measured daily. Green line, FGF2 + PDGF-BB; red line, FGF2; blue line, vector + FGF2; purple line, vector and black PDGF-BB. (B) At day 13 after tumor cell implantation, tumors were removed and stained with an anti-CD31 antibody, and tumor blood vessels were analyzed by confocal microscopy using 3D projections. GFP-expressing tumor cells are green in color, and tumor blood vessels are presented in red. Arrows point to tip-cell sprouts from the vascular plexuses induced by FGF2 and PDGF-BB. (C) Quantification of CD31-positive tumor vessels from 8-12 randomized cryosectioned fields. The data represent means of average determinants $\pm \mathrm{SEM}$. ${ }^{*} P<0.05$; ${ }^{* * *} P<0.001$. Scale bar: $100 \mu \mathrm{m}$ (B, upper and lower panels); $50 \mu \mathrm{m}$ (B, middle panels). V, vector.

cular cells in promoting tumor neovascularization, vessel maturation, and metastasis. FGF2 triggers PDGF-BB responses in ECs and enhances PDGF-BB-induced tumor angiogenesis. Conversely, PDGF-BB augments the effect of FGF2 on VSMCs. The underlying mechanisms of this reciprocal interaction involve transcriptional upregulation of their receptors on ECs and VSMCs. Activation of this interactive loop in a tumor environment leads to accelerated but uncoupled growth of ECs and VSMCs, leading to the formation of primitive tumor vasculature, which promotes metastasis.

PDGF-BB is a well-characterized growth factor displaying potent biological effects on mural cells including pericytes and VSMCs, but not on ECs $(21,23,24)$. Inactivation of both PDGF-B and PDGFR- $\beta$ genes results in lethal embryonic phenotypes of improper development of the vasculature due to lack of pericytes and VSMCs $(23,24)$. Although a recent report demonstrates that PDGFR- $\alpha$ but not $-\beta$ is expressed in isolated ECs, it is not known how PDGFR- $\alpha$ expression is regulated or what its role is in mediating PDGF-BB-induced angiogenic responses (32). All members of the PDGF family display potent angiogenic activity in vivo (33). For example, PDGF-AA, PDGF-AB, PDGF-BB, and PDGF-CC potently induce neovascularization in mouse corneas. If VSMCs are the primary vascular target cells for PDGFs, how could they induce the growth of angiogenic vessels consisting mainly of ECs? These and other in vivo studies suggest that the endothelial effects of PDGFs are probably modulated by other factors. In the present study, we provide compelling in vitro and in vivo evidence that FGF2 induces promoter activities of both PDGFR- $\alpha$ and PDGFR- $\beta$ in ECs and the increased transcripts lead to high levels of PDGFR protein expression in ECs. When high levels of PDGFR- $\alpha$ and PDGFR- $\beta$ in ECs become activated by available ligands, the PDGF-induced angiogenic response is overwhelming. 
A
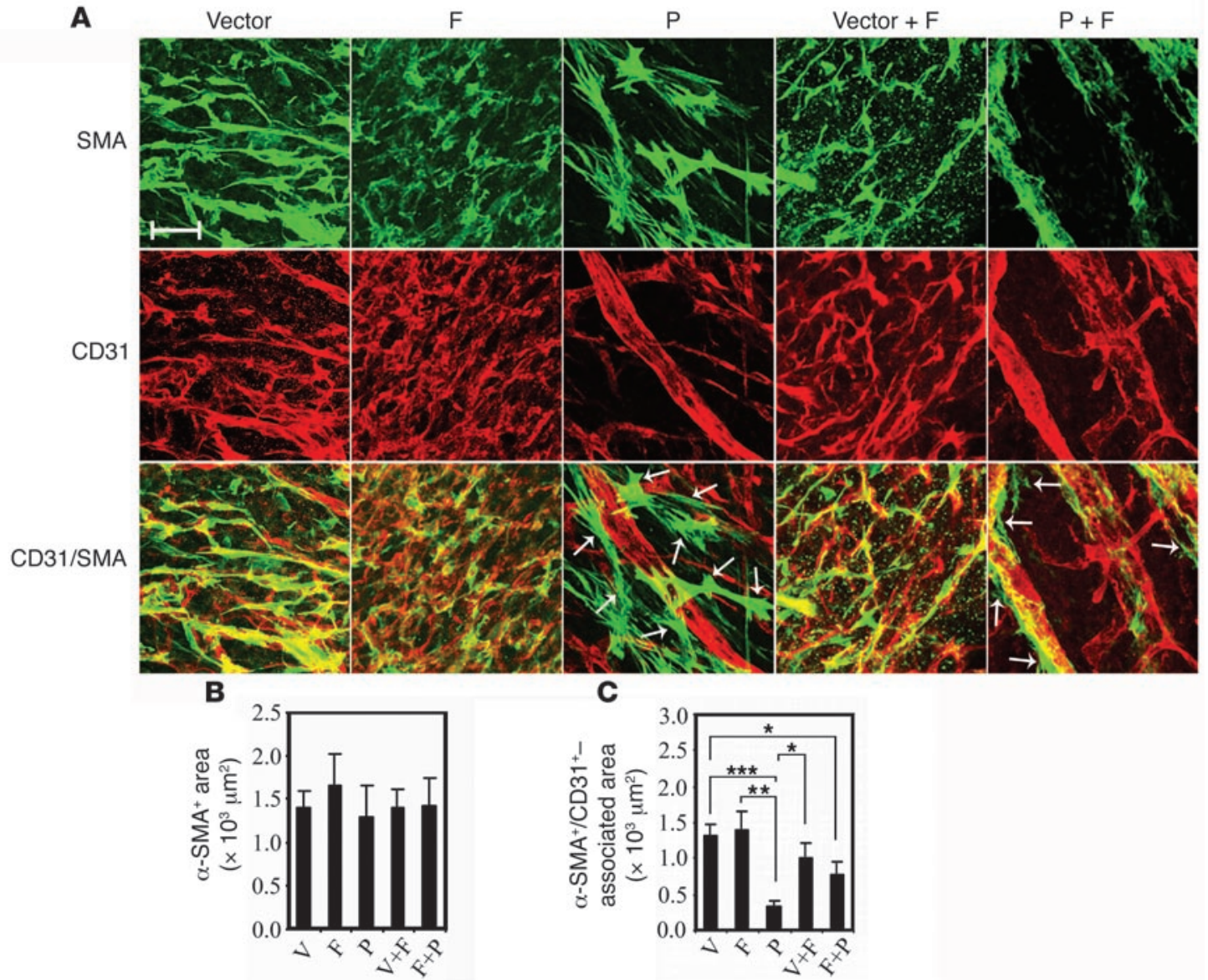

\section{Figure 6}

Interaction between VSMCs and ECs in tumors. At day 14 after implantation, tumor tissues were double-stained with an anti-CD31 antibody and an anti- $\alpha$-SMA antibody. (A) The CD31- (red) and $\alpha$-SMA-positive (green) signals were revealed by Alexa Fluor 555- and Alexa Fluor 647-labeled antibodies, respectively, using single-layer projections in a confocal microscope. Overlapping double-positive signals are in yellow color. (B and C) Total numbers of $\alpha$-SMA-positive vessels were randomly counted from 12 fields/group, and percentages of $\alpha$-SMA-positive vessels versus total CD31-positive vessels were calculated. The data represent means of average determinants \pm SEM. ${ }^{*} P<0.05$; ${ }^{* \star} P<0.01$; ${ }^{\star * *} P<0.001$. Scale bar: $50 \mu \mathrm{m}$.

FGF2 potently stimulates EC proliferation but has almost no effect on chemotaxis (30). Additionally, our present study shows that PDGF-BB is able to induce EC migration but not proliferation. Angiogenesis requires both EC proliferation and migration, and FGF2 triggered PDGF response in ECs, probably to compensate for its defective effect on cell migration. When both systems became activated, coordinated EC proliferation and migration contributed to guided vessel growth. Increased levels of PDGF-BB positively fed back to the FGF system in VSMCs by transcriptional upregulation of FGFR1 expression (Figure 9). This result is consistent with previous findings that PDGF-BB upregulates FGFR1 expression in VSMCs (34). PDGF-BB only facilitates FGF2-induced VSMC proliferation but not migration. In a similar scenario as for ECs, PDGF-BB cross-communicates with FGF2 to keep a balance between VSMC proliferation and migration. However, the overall coordinated control mechanism of EC and VSMC growth and association in newly formed blood vessels is not understood. Our findings uncover the underlying molecular mechanisms by which FGF2 and PDGF-BB synergistically and coordinately stimulate neovascularization. The angiogenic synergism is particularly limited to the interactive loop between FGF and PDGF systems because other potent angiogenic factors such as VEGF-A are unable to induce PDGFR expression and promote synergistic angiogenesis in vitro and in vivo (31). There seems to be a functional difference of FGF2 plus PDGF-BB-induced vasculatures in the tumor versus healthy tissue environments. In the ischemic muscle tissue, delivery of dual factors induced functional and relatively mature blood vessels, which improved functional outcomes of ischemic tissues. However, in the tumor environment, FGF2 plus PDGF-BB promoted relatively premature vasculatures that mediated metastasis. Although it is unclear why these 2 factors induced premature blood vessels in tumors, it is possible that other factors such as VEGF-A could also play a role in a further complex interplay between different factors. For example, it is known that FGF2 and VEGF-A could also synergistically induce angiogenesis (35). The other possibility is that tumors constitutively produce these factors at high levels, whereas a slow-release system is used in the ischemic settings. The third possibility is that PDGF-BB released by a slow-release scaffold could build up a growth factor-release gradient and attract vessel growth and mural cell recruitments toward the gradient. In contrast, the tumor-released PDGF-BB is evenly 

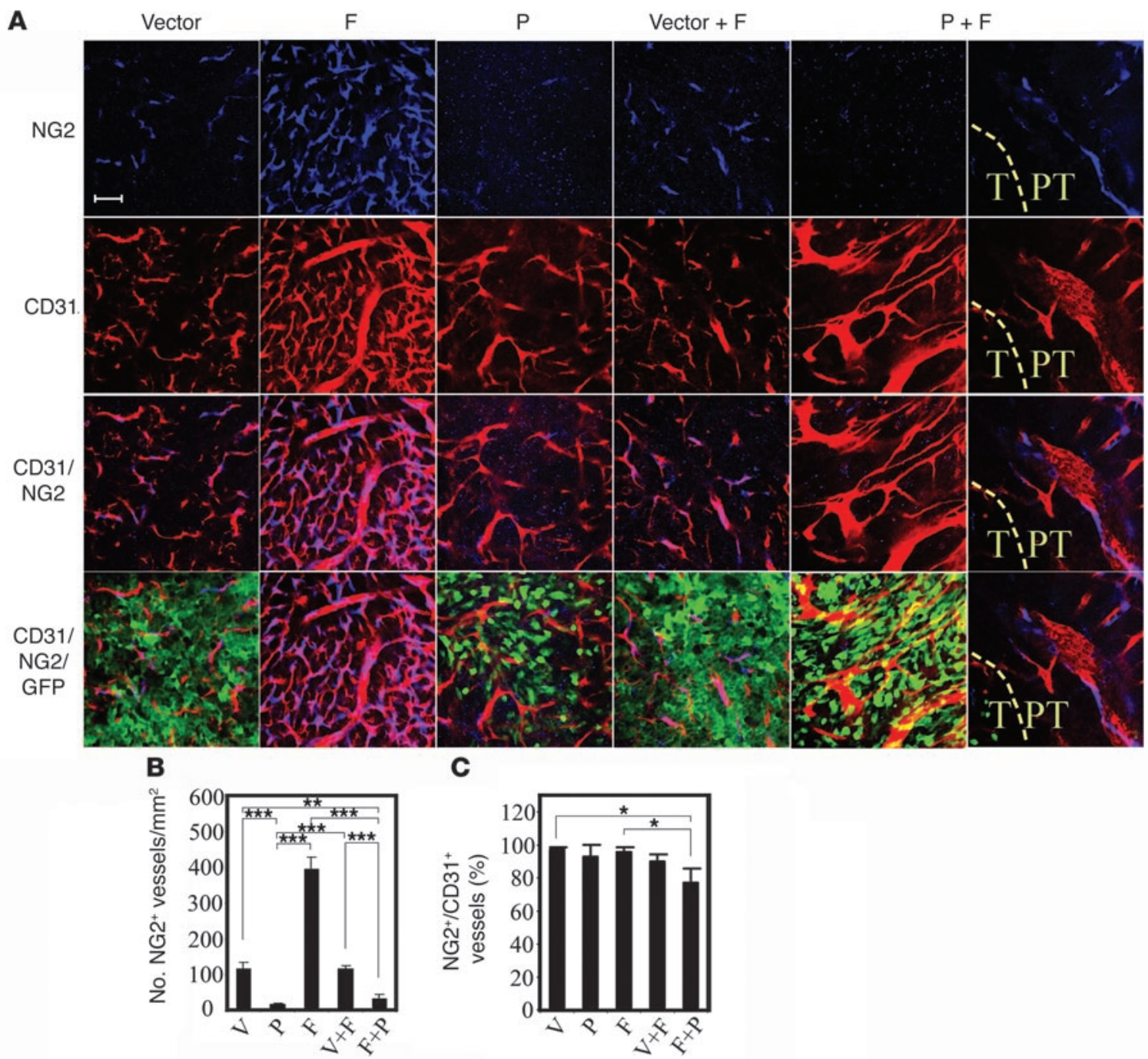

Figure 7

Interaction between pericytes and ECs in tumors. At day 10 after implantation, tumor tissues were double-stained with an anti-CD31 antibody and an anti-NG2 antibody. (A) The CD31- (red) and NG2-positive (blue) signals were revealed by Alexa Fluor 555- and Cy5-labeled antibodies, respectively, using single-layer projections in a confocal microscope. Tumor cells were GFP positive (green). T, Intratumoral area; PT, peritumoral area. (B) Total numbers of NG2 positive vessels were randomly counted from 9 fields/group, and (C) percentages of NG2-positive vessels relative to total CD31-positive vessels were calculated. The data represent means of average determinants \pm SEM. ${ }^{*} P<0.05$; ${ }^{* *} P<0.01$; ${ }^{* * *} P<0.001$. Scale bar: $50 \mu \mathrm{m}$.

distributed within the tumor tissue and lacks such gradient effect. These hypotheses warrant further investigation.

One of the most intriguing findings in our present study is that the tumor-produced PDGF-BB promotes disassociation of VSMCs from the tumor vasculature. This finding contradicts the known effect of PDGF-BB on recruitment of VSMCs into the newly formed blood vessels. Why does PDGF-BB repel VSMCs from tumor vessels? What is so special about the tumor environment? Although these questions may be involved in complex genetic and epigenetic mechanisms of many molecular players that regulate tumor growth and vessel formation, a simple and straightforward answer could be implicated in a spatiotemporal relationship between the site of PDGF-BB production and tumor vessel development. In most other studies, PDGF-BB is produced by growing ECs, which play guiding and recruiting roles in the growing cone of vessel tips for attract- ing pericytes and VSMCs. However, when PDGF-BB is produced by tumor cells, VSMCs and pericytes might become "confused" about their migration and lose their guidance movement due to lack of chemoattractant gradient. Thus they could move away from blood vessels and remain in close contact with tumor cells. In addition to VSMCs, ECs might also become confused about their migration, as their tip cells are randomly distributed in the vascular plexuses (Figure 5B). The underlying mechanism of the inhibitory effect of PDGF-BB on recruitment of pericytes into the tumor vasculature remains unknown. These findings are crucially important for understanding the role of PDGFs in tumor vessel maturation, patterning, and drug target definition.

Bloodstream metastasis is dependent not only on vessel density but also on quality of vascular structure. Primitive tumor vessels are vulnerable for tumor cell invasion and provide a structural basis 

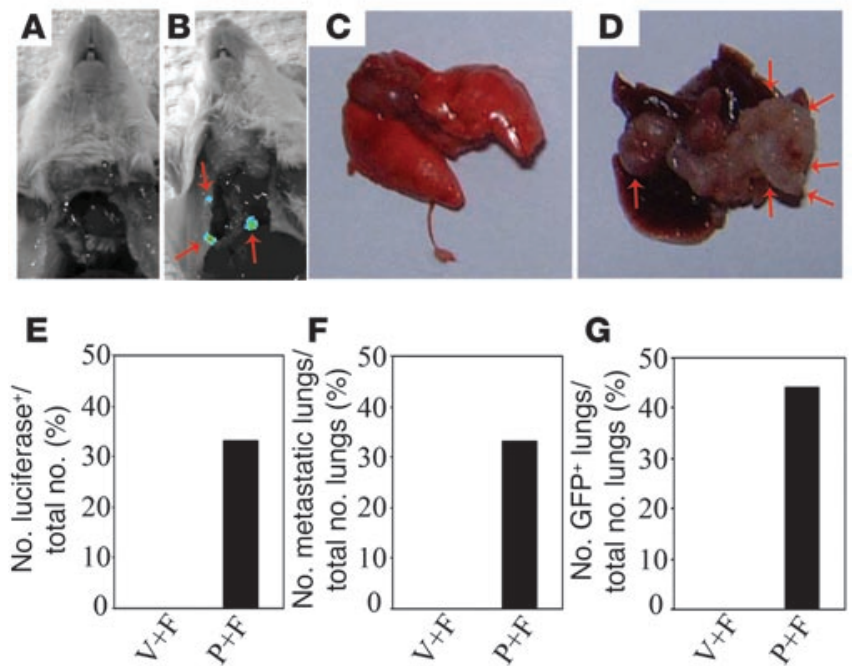

G

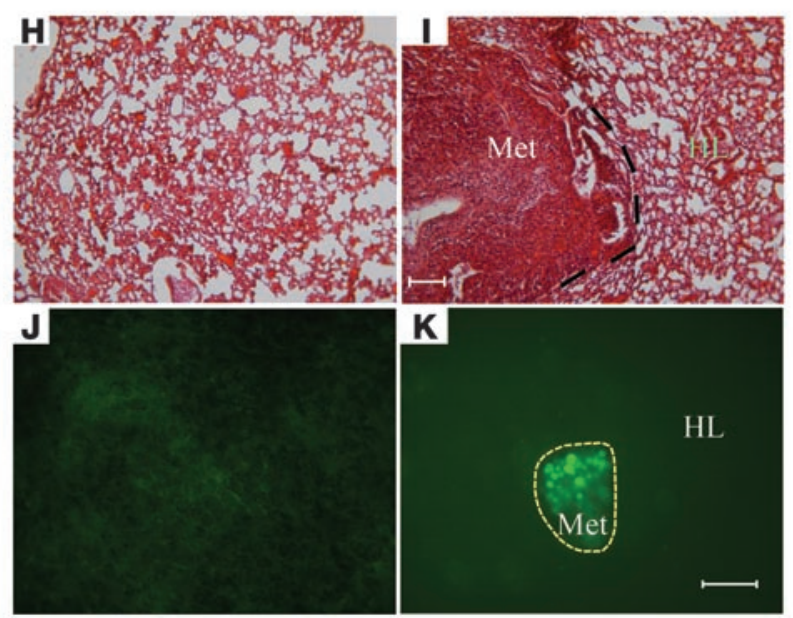

for metastasis. In theory, PDGF-BB would promote maturation of tumor vessels by recruiting VSMCs to the nascent vasculature and thus prevent metastasis. However, in the presence of FGF2, PDGF-BB not only acts on VSMCs but also on ECs, leading to uncoupling of growth of both compartments and the formation of a primitive nascent vasculature, which mediates pulmonary metastasis of less invasive FGF2-expressing tumor cells. We should emphasize that T241 fibrosarcoma cells express very low levels of PDGFRs and lack response to PDGF-BB stimulation (ref. 36 and our unpublished observations). Thus stimulation of pulmonary metastasis by the FGF2/PDGF-BB interactive loop is unlikely due to their direct effects on tumor cells. The fact that both PDGF-BB and FGF2 tumor cells gain a metastatic feature in mice with co-implanted tumors further suggests that a common mechanism is likely responsible for tumor spread. Indeed, implantation of a mixture of FGF2 plus vector tumor cells does not give rise to metastasis. These findings support the notion that reciprocal vascular interactions between FGF2 and PDGF-BB might be responsible for promoting lung metastasis.

Although VEGF-A-mediated vascular functions have become attractive targets for antiangiogenic cancer therapy, long-term delivery of anti-VEGF-A agents in a spontaneous mouse tumor model results in drug resistance, suggesting that tumors switch to other angiogenic factors (19). From the mouse tumors, one would

\section{Figure 8}

Stimulation of metastasis by coexpression of FGF2 and PDGF-BB in tumors. Subcutaneous tumors were removed when they reached $1.5 \mathrm{~cm}^{3}$. (A and B) Mice were followed for 4 weeks, and pulmonary metastases of vector/FGF2 tumor-bearing (A) and PDGF-BB/FGF2 tumor-bearing (B) mice were examined by bioluminescence. Arrows point to luciferase-positive lung metastases. (E) Numbers of pulmonary luciferase-positive animals relative to total numbers of animals are presented as percentages. (C and $\mathbf{D})$ Examples of lung morphology of vector/FGF2 tumor-bearing (C) and PDGF-BB/FGF2 tumorbearing (D) mice and arrows in $D$ panel point to visible lung metastases in the PDGF-BB/FGF2 group. (F) Numbers of lungs with visible pulmonary metastases versus total numbers of lungs are presented as percentages. ( $\mathbf{H}$ and $\mathbf{I}$ ) Lung tissues were stained with $\mathrm{H} \& \mathrm{E}$ and metastatic nodules were validated in the PDGF-BB/FGF2 group (I). (H) No metastasis was visible in the vector/FGF2 tumor-bearing control group. ( $\mathbf{J}$ and $\mathbf{K}$ ) GFP-positive metastases were revealed by analysis of lung sections under a fluorescent microscope of vector/FGF2- (J) and PDGF-BB/FGF2-tumor bearing (K) mice. (G) Numbers of lungs with GFP-positive metastases relative to total numbers of lungs are presented as percentages. Met, metastasis; $\mathrm{HL}$, healthy lung tissue. Scale bars: $100 \mu \mathrm{m}$.

expect a similar drug-resistant mechanism to also exist in cancer patients. PDGFs are attractive targets for cancer therapy, and antiPDGF agents are effective for the treatment of experimental and human tumors (37). Paradoxically, PDGF and probably other vascular remodeling factors could play an important role in normalization of tumor vasculature, which might increase chemotherapeutic drug delivery $(14,38)$. This paradoxical issue needs to be further clarified in future studies. Although PDGF-BB and FGF2 may be expressed at low levels in some tumors, their coordinated activity in the promotion of synergistic angiogenesis and uncoupling of vascular remodeling in a tumor environment should not be underestimated for tumor growth and metastasis.

Taken together, our work provides what we believe to be a novel mechanistic insight on transcriptional regulation of angiogenic responses and vascular remodeling induced by FGF2 and PDGF-BB. To our knowledge, this is the first report describing how the interplay between different angiogenic factors can promote tumor neovascularization and metastasis. As tumor blood vessels are constantly exposed to various angiogenic factors, understanding molecular mechanisms of interplays between various angiogenic factors is crucial for the development of effective therapeutic agents. Our findings provide one such example of reciprocal interactions and a potentially novel therapeutic option.

\section{Methods}

Reagents and animals. The antibodies used in our studies include a rat antimouse CD31 monoclonal antibody (BD - Pharmingen), a mouse antihuman $\alpha$-SMA (Dako), and a rabbit anti-mouse NG2 antibody (Chemicon International). Immunoincompetent 6- to 8-week-old female SCID mice were acclimated and caged in groups of 6 or less. Animals were followed up to 3-6 weeks and anesthetized by an injection of a mixture of Hypnorm (fentanyl citrate, fluanisone; VetaPharma) and Midazolam (dormicum; Roche) (1:1) before all procedures and sacrificed by a lethal dose of $\mathrm{CO}_{2}$, followed by cervical dislocation. All animal studies were reviewed and approved by the animal care and use committee of the North Stockholm Animal Board (Stockholm, Sweden).

EC proliferation assay. A 72-hour BCE cell proliferation assay was performed as previously described (39). Cells growing in gelatinized 6-well plates were 


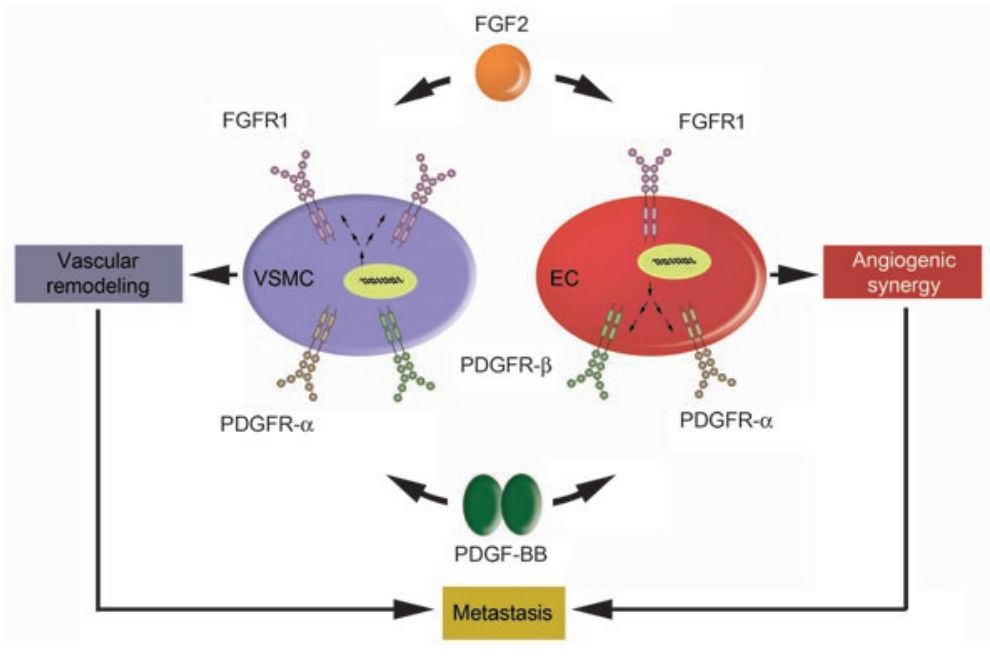

Figure 9

Schematic representation of reciprocal interplay between FGF2 and PDGF-BB in promoting tumor angiogenesis and metastasis. FGF2 induces expression of PDGFR- $\alpha$ and $-\beta$ in ECs, which are activated by PDGF-BB. Activation of PDGFRs leads to enhanced EC migration, which would synergistically coordinate with FGF2-induced EC proliferation. Both proliferation and migration of ECs are essential steps of tumor angiogenesis and are manifested in vivo as angiogenic synergism, which promotes metastasis. Conversely, PDGF-BB upregulates FGFR1 expression in VSMCs, which mediates proliferation signals triggered by FGF2. The FGF2-induced VSMC proliferation synergistically cooperates with PDGF-BBinduced VSMC proliferation and migration to crosstalk to nascent vasculature for remodeling. The coordination between synergistic angiogenesis and synergistic vascular remodeling in facilitating a functional vasculature remains unknown. In the tumor environment, it appears that FGF2/PDGF-BB-induced synergistic angiogenesis is not well coordinated with the angiogenic effects of FGF2 and PDGF-BB vascular remodeling. As a consequence, these 2 factors induce disorganized vasculatures and promote cancer metastasis.

dispersed in $0.25 \%$ trypsin solution and resuspended with DMEM containing $0.5 \%$ FCS. We added $1 \times 10^{4}$ cells to each gelatinized well of 24 -well plates and incubated them at $37^{\circ} \mathrm{C}$ for 1 hour, followed by addition of various concentrations of FGF2 and PDGF-BB in quadruplicates to each well and further incubation for 12 hours. The old medium was replaced with fresh medium in the presence or absence of growth factors $(10 \mathrm{ng} / \mathrm{ml} \mathrm{FGF} 2$ or $100 \mathrm{ng} / \mathrm{ml}$ PDGF-BB) and incubated for 60 hours. After a total of 72 hours of incubation, cells were trypsinized, resuspended in Isoton II solution (Beckman Coulter), and counted with a Coulter counter (Beckman Coulter).

VSMC proliferation. VSMCs were isolated from the aortic media of male F344 rats by collagenase digestion and cultured in F-12 Ham medium (GIBCO) supplemented with $50 \mu \mathrm{g} / \mathrm{ml}$ L-ascorbic acid, $50 \mu \mathrm{g} / \mathrm{ml}$ streptomycin, $50 \mathrm{IU} / \mathrm{ml}$ penicillin, and 20\% FCS (GIBCO) as previously described (40). These cells were kindly provided by Eric Wahlberg's laboratory at the Karolinska Hospital, Stockholm, Sweden. Cells growing in 6-well plates were dispersed in a trypsin solution $(0.25 \%)$ containing $10 \mathrm{mM}$ EDTA and were resuspended with F-12 Ham medium containing $0.5 \%$ FCS. Cells $\left(10^{4}\right)$ were added to each well of 24 -well plates and incubated at $37^{\circ} \mathrm{C}$ for 3 hours, followed by addition of various concentrations of FGF2 and PDGF-BB in quadruplicates and were further incubated for 12 hours. Old medium was replaced with fresh medium in the presence or absence of growth factors (10 ng/ml FGF2 or $100 \mathrm{ng} / \mathrm{ml}$ PDGF-BB) and incubated for 60 hours. After a total of 72 hours of incubation, cells were trypsinized, resuspended in Isoton II solution (Beckman Coulter), and counted with a Coulter counter.

EC and VSMC chemotaxis assay. The motility responses of BCE or VSMC cells to FGF2 and PDGF-BB were assayed by using the modified Boyden chamber technique described in ref. 13. Briefly, the ability of $\mathrm{BCE}$ or VSMC cells to migrate through a micropore nitrocellulose filter ( $8 \mu \mathrm{m}$ pore size) was measured as a criterion for chemotactic stimuli. Cells were either pretreated or not pretreated with $10 \mathrm{ng} / \mathrm{ml} \mathrm{FGF2}$ or $100 \mathrm{ng} / \mathrm{ml}$ PDGF-BB for 24 hours, and cells were trypsinized and resuspended in fresh medium supplemented with $0.5 \%$ FCS. Approximately $5 \times 10^{4}$ cells were added into each of the upper wells of the Boyden chamber (48-well chamber), which contained $10 \mathrm{ng} / \mathrm{ml}$ of FGF2 or $100 \mathrm{ng} / \mathrm{ml}$ of PDGF-BB in the lower chamber. The cells were incubated for 4 hours at $37^{\circ} \mathrm{C}$, after which the Boyden chamber was disassembled and cells attached to the filter were fixed in methanol and stained with a Giemsa solution. Four replicate samples were used in each experiment, and all experiments were performed at least 4 times. Cells that had migrated through the filter were counted using a light microscope and plotted as numbers of migrating cells per optic field.

$A c-L D L$ and $\alpha$-SMA staining of VSMCs and ECs. VSMCs and ECs were grown on coverslips in 6-well culture dishes under growth conditions described above. Cells in approximately $80 \%$ confluency were fixed in acetone for 20 minutes and vigorously washed with PBS prior to incubation with an $\alpha$-SMA antibody (1:1000 dilution). An anti-mouse IgG-FITC-labeled antibody was used as the secondary antibody (Vector Laboratories). Ac-LDL labeled with the fluorescent dye Dil was diluted to $10 \mu \mathrm{g} / \mathrm{ml}$ in DMEM supplemented with $10 \%$ FCS. Medium was then added to 6-well plates, where VSMCs or ECs were growing on coverslips as described above and incubated for 4 hours at $37^{\circ} \mathrm{C}$. Media was removed from the culture, and cells were washed several times with probe-free media and examined under a fluorescent microscope.

Transient transfection and reporter gene assay. PDGFR- $\alpha$ and PDGFR- $\beta$ promoter-luciferase fusion gene constructs were generated as previously described (41-43). BCE-human telomerase reverse transcriptase-positive $\left(\mathrm{BCE}-\mathrm{hTERT}^{+}\right.$) cells were maintained in DMEM containing 10\% FBS, $100 \mathrm{U} / \mathrm{ml}$ penicillin, and $100 \mu \mathrm{g} / \mathrm{ml}$ streptomycin. About $1 \times 10^{5}$ cells were seeded in each well of 12 -well plates for 16 hours prior to transfection, yielding approximately $95 \%$ of confluency. Before transfection, the medium was changed into serum-free and streptomycin/penicillin-free DMEM. The cells were then transfected with FuGENE6 (Roche) reagent according to the manufacturer's protocol. Briefly, $1.2 \mu \mathrm{l} \mathrm{FuGENE6} \mathrm{reagent} \mathrm{was} \mathrm{diluted} \mathrm{in} 100 \mu \mathrm{l}$ serum-free and penicillin/ streptomycin-free DMEM, and then 350 ng PDGFR- $\alpha$ and PDGFR- $\beta$ promoter-reporter gene constructs and $50 \mathrm{ng}$ CMV- $\beta$-galactosidase expression vector were added. These optimal values were determined experimentally several times. The resulting complex was added to BCE or VSMC cells, and $10 \mathrm{ng} / \mathrm{ml}$ FGF2 or PDGF-BB were added. Cells were incubated for 40 hours, and luciferase activity was determined at the end of incubation according to the protocol described in the Bright-Glo luciferase kit (Promega). Measurements were done in quadruplicate using a Sirius luminometer (Berthold).

The FGFR1-CAT was kindly provided by J. DiMario, Rosalind Franklin University, Chicago, Illinois, USA. The promoter-reporter fusion gene constructs were used for transfection of BCE and VSMCs according the procedures described above. The transfected cells were treated with FGF2 or PDGF-BB as described above. For measurement of CAT activity, a standard protocol provided by the manufacturer was used (Roche).

RT-PCR. A quantitative RT-PCR method was performed to amplify PDGFR- $\alpha$ and PDGFR- $\beta$ using cDNAs prepared from BCE cells as templates. The following primers were used: a 27 -mer $5^{\prime}$-end nucleotide sequence for PDGFR- $\alpha$ (5'-GGCCTGACTTTGTTGGATTTGTTGAGC- 
$3^{\prime}$ ), a 26-mer $3^{\prime}$-end nucleotide sequence for PDGFR- $\alpha$ (5'-CGTTGCGAGCCGCCAGGTCCCGATGG-3'), a 21-mer 5 '-end nucleotide sequence for PDGFR- $\beta$ 5'-GCAGACTGTGGTCCGCCAAGG-3', and a 24 -mer $3^{\prime}$-end nucleotide sequence for PDGFR- $\beta$ 5'-CGACCACGGTGACATTTGATAGCC-3'. As a loading control bovine GAPDH cDNA was used, and paired primers of a 29-mer $5^{\prime}$-end nucleotide sequence for bovine GAPDH (5'-CGGAGTGAACGGATTCGGCCGCATCGGGC-3') and a 30-mer 3'end nucleotide sequence for bovine GAPDH (5'-CCCAGCCTTCTCCATGGTAGTGAAGACCCC-3') were used. The PCR-amplification program consisted of 35 cycles of the following: $94^{\circ} \mathrm{C}$ for 2 minutes, $50^{\circ} \mathrm{C}$ for 2 minutes, and $72^{\circ} \mathrm{C}$ for 2 minutes.

Mouse corneal micropocket assay. The mouse corneal assay was performed as previously described (28). Micropellets containing $40 \mathrm{ng}$ of FGF2 (Pharmacia \& Upjohn), 160 ng of PDGF-BB (Peprotech Inc.), or 40 ng of FGF2 plus 160 ng of PDGF-BB were implanted into mouse corneal micropockets. Circumferential neovascularization and vascularization areas were measured at various time points.

In situ hybridization. We used 2 probes complementary to PDGFR- $\alpha$ (nucleotides 423-470 and 3083-3130) and 2 probes complementary to PDGFR- $\beta$ (nucleotides 946-996 and 2610-2657). All probes were used separately and did not match any known sequence in GenBank except those of the intended genes. Corneal histologic sections were hybridized with the ${ }^{33}$ P-labeled probes. Slides were rinsed, dehydrated, and emulsion dipped. After 5 weeks of exposure, slides were developed and counterstained with cresyl violet. Specific labeling was confirmed by similar expression patterns revealed by 2 probes each (complementary to different parts of the mRNA) for PDGFR- $\alpha$ and PDGFR- $\beta$. See Supplemental Methods for details and quantification of autoradiographic signals (supplemental material available online with this article; doi:10.1172/JCI32479DS1).

Immunoblot analysis. Monolayers of BCE cells were stimulated with $10 \mathrm{ng} / \mathrm{ml} \mathrm{FGF} 2$ or $100 \mathrm{ng} / \mathrm{ml}$ PDGF-BB for various time points. After extensive washing with PBS, cells were lysed with a lysis buffer of $20 \mathrm{mM}$ Hepes, $\mathrm{pH}$ 7.1, $150 \mathrm{mM} \mathrm{NaCl}, 50 \mathrm{mM} \mathrm{NaF}, 1 \%$ Triton X-100, $30 \mathrm{mM} \mathrm{Na}_{4} \mathrm{P}_{2} \mathrm{O}_{7}$, $5 \mu \mathrm{M} \mathrm{ZnCl}_{2}, 20 \mathrm{mM} \beta$-glycerophosphate, $10 \mathrm{mM}$ 4-nitrophenyl phosphate, $1 \mathrm{mM} \mathrm{DTT}, 100 \mu \mathrm{M} \mathrm{Na}_{3} \mathrm{VO}_{4}$, and $0.5 \mathrm{mM} \mathrm{PMSF}$. Cell lysates were centrifuged in a tabletop centrifuge at the maximum speed $(18,000 \mathrm{~g})$ for 20 minutes, and the supernatants were collected for protein determination by using a modified Lowry method (Bio-Rad) with BSA as a standard. Equal amounts of each sample were loaded onto $4 \%-20 \%$ acrylamide SDS/PAGE gradient gels (NOVEX). Proteins were transferred onto nitrocellulose membranes (Schleicher \& Schuell), which were subsequently blocked overnight at room temperature with $2.5 \%$ BSA-PBST $(140 \mathrm{mM} \mathrm{NaCl}, 2.7 \mathrm{mM} \mathrm{KCl}$, $8.1 \mathrm{mM} \mathrm{Na}_{2} \mathrm{HPO}_{4}, \mathrm{pH} 7.3$, and $0.3 \%$ Triton X-100), followed by incubation at $4{ }^{\circ} \mathrm{C}$ overnight with primary antibodies against PLC $\gamma-1$ (kindly provided by Carl-Henrik Heldin, Uppsala, Sweden; ref. 44), including phosphospecific Src (PY418; Biosource), phosphospecific-Akt (Ser473; Cell Signaling Technology), phosphospecific-p44/p42 MAPK (Thr202/Tyr204; Cell Signaling Technology), phosphospecific p38 MAPK (The180/Tyr182; Cell Signaling Technology), and phosphospecific SAPK/JNK (Thr183/Tyr185; Cell Signaling Technology) antibodies. After extensive washing with PBST, membranes were incubated for about 1 hour at room temperature with peroxidase-coupled secondary antibodies. After further washing, immunoreactive signals were revealed by chemiluminescence. We used an antibody specific for $\beta$-actin (monoclonal anti- $\beta$-actin, clone AC- 15 [mouse ascites fluid, Mouse IgG1]; Sigma-Aldrich) as a loading control.

Tumor growth assay. A murine fibrosarcoma T241 cell line was used for the generation of transfectant cell lines overexpressing enhanced GFP and hPDGF-BB, or the empty vector as previously described (45). The fibrosarcoma cells expressing a secreted form of hFGF2 were established as previously described (46). In some experiments, vector and FGF2 tumor cells were further stably transduced with the luciferase gene as previously reported (47). Approximately $0.5 \times 10^{6}$ vector-, FGF2-, and PDGF-BBtransduced tumor cells; $0.25 \times 10^{6}$ vector-transduced plus $0.25 \times 10^{6} \mathrm{FGF} 2$ transduced tumor cells; or $0.25 \times 10^{6} \mathrm{PDGF}-\mathrm{BB}$-transduced plus $0.25 \times 10^{6}$ FGF2-transduced tumor cells were subcutaneously implanted on the back of each 6- to 8-week-old female SCID mouse, and tumor volumes were measured as previously reported $(39,48)$.

Metastasis assay. When primary tumors reached a volume of $1.5 \mathrm{~cm}^{3}$ (the ethical limit), primary tumors were surgically removed under anesthetic conditions ( $n=10$ /group). The open wounds were sutured, and mice were observed for 4 weeks. Mice were i.p. injected with $1.5 \mathrm{mg} / 200 \mu \mathrm{l}$ of D-luciferin (Xenogen) and scanned for luciferase-positive metastases by bioluminescence imaging analysis (IVIS 100; Xenogen). The mice were sacrificed at end of week 4 after removal of the primary tumors. After opening the chest and abdomen, the exposed organs and tissues were further scanned for luciferase-positive metastases. Lungs were resected, and surface metastases were examined visually under a light microscopy. Several organs including lung, liver, spleen, kidney, ovary, and brain were sectioned and analyzed for GFP-positive tumor metastases under a fluorescent microscope.

Histology and whole-mount staining. Malignant and nonmalignant paraffin-embedded tissues were sectioned at $5 \mu \mathrm{m}$ thickness and stained with H\&E according to our previously described methods (13). Whole-mount staining was performed according to previously published methods (45). Briefly, small pieces of tissues were cut into thin slices and fixed in 3\% PFA overnight, followed by treatment with proteinase $\mathrm{K}(20 \mu \mathrm{g} / \mathrm{ml})$. Rat anti-mouse CD31 antibodies were used as primary antibodies and goat anti-rat Alexa Fluor 555-conjugated antibodies (Invitrogen) were used as secondary antibodies. Additionally, a mouse anti-human $\alpha$-SMA primary antibody (Dako) and a rabbit anti-mouse secondary antibody Alexa Fluor 647-conjugated antibody were used (Invitrogen). An anti-NG2 antibody (Chemicon) was used for detection of pericytes. Slides were examined under a confocal microscope (Zeiss Confocal LSM510 microscope). By scanning 10 thin sections of each sample (4-5 $\mu \mathrm{m}$ long), 3D images of each tissue sample were assembled. Quantitative analysis from at least 5 different tissue sections was performed using the color range tool of Adobe Photoshop CS software program. Alternatively, slides were examined using a custom-built dual-mode Ultraview LCI confocal system (PerkinElmer) at the Karolinska Institute visualization core facility. 3D reconstitution was carried out using ImageJ, and images were further processed in Adobe Photoshop CS.

Immunofluorescent staining. Cryostat tissue sections $20 \mu \mathrm{m}$ thick were incubated with a specific antibody against CD31 according to standard immunohistochemical procedures. Briefly, after 3 washes in PBS, specimens were incubated for 30 minutes in a blocking solution containing $4 \%$ nonimmune goat serum (Vector Laboratories) in PBS, followed by incubation with primary antibodies for 2 hours at room temperature. After 3 rigorous washes with PBS, tissues were incubated for 1 hour at room temperature with secondary antibodies labeled with Alexa Fluor 555-conjugated red (1:500). Sections were mounted on glass slides with Vectashield mounting medium (Vector Laboratories). Positive signals were photographed under a fluorescent microscope $(\times 20$ objective magnification, Zeiss Confocal LSM510 Microscope). Quantitative analyses were obtained from at least 3 sections of 3 different animals.

Statistics. Statistical analyses of in vitro and in vivo results were performed using the standard 2-tailed Students $t$ test on Microsoft Excel. Some experimental data were analyzed using the 2-way ANOVA method in Microsoft Excel, followed by a Bonferroni post hoc test using the GraphPad QuickCalcs online Web site (http://www.graphpad.com/quickcalcs/posttest1.cfm). $P<0.05, P<0.01$, and $P<0.001$ were deemed as significant, highly significant, and extremely significant, respectively. 


\section{Acknowledgments}

We thank Ina Rostalski for her technical assistance in carrying out the smooth muscle cell chemotaxis experiments. We thank David Ornitz at the Washington University School of Medicine (St. Louis, Missouri, USA), Joe DiMario at the Rosalind Franklin University (Chicago, Illinois, USA), and S. Ezzat, Mount Sinai and University of Toronto (Toronto, Ontario, Canada), for FGFR reporter gene constructs. We also thank Lasse Dahl Ejby Jensen and Yuan Xue for technical assistance. We thank Lilly Wu and Jeremy Burton at the UCLA Medical School for labeling tumor cells with luciferase. This work was supported by the laboratory of Y. Cao through research grants from the Swedish Research Council, the Swedish Heart and
Lung Foundation, the Swedish Cancer Foundation, the Center for Gender Medicine, the Karolinska Institute Foundation, and the Torsten and Ragnar Söderberg's Foundation and by European Union Integrated Projects of Angiotargeting Contract 504743 (to Y. Cao) and VascuPlug Contract STRP 013811 (to Y. Cao).

Received for publication April 23, 2007, and accepted in revised form July 25, 2007.

Address correspondence to: Yihai Cao, Microbiology and Tumor Biology Center, Karolinska Institutet, 17177 Stockholm, Sweden. Phone: 46-8-5248-7596; Fax: 46-8-31-94-70; E-mail: yihai.cao@mtc.ki.se.
1. Carmeliet, P., and Jain, R.K. 2000. Angiogenesis in cancer and other diseases. Nature. 407:249-257.

2. Ellis, L.M., and Fidler, I.J. 1996. Angiogenesis and metastasis. Eur. J. Cancer. 32A:2451-2460.

3. Ferrara, N., and Kerbel, R.S. 2005. Angiogenesis as a therapeutic target. Nature. 438:967-974.

4. Folkman, J. 1971. Tumor angiogenesis: therapeutic implications. N. Engl. J. Med. 285:1182-1186.

5. Hanahan, D., and Folkman, J. 1996. Patterns and emerging mechanisms of the angiogenic switch during tumorigenesis. Cell. 86:353-364.

6. Kaplan, R.N., et al. 2005. VEGFR1-positive haematopoietic bone marrow progenitors initiate the premetastatic niche. Nature. 438:820-827.

7. Kerbel, R.S. 2006. Antiangiogenic therapy: a universal chemosensitization strategy for cancer? Science. 312:1171-1175.

8. Li, J.L., and Harris, A.L. 2005. Notch signaling from tumor cells: a new mechanism of angiogenesis. Cancer Cell. 8:1-3.

9. McDonald, D.M., and Choyke, P.L. 2003. Imaging of angiogenesis: from microscope to clinic. Nat. Med. 9:713-725.

10. Noguera-Troise, I., et al. 2006. Blockade of Dll4 inhibits tumour growth by promoting non-productive angiogenesis. Nature. 444:1032-1037.

11. Ridgway, J., et al. 2006. Inhibition of Dll4 signalling inhibits tumour growth by deregulating angiogenesis. Nature. 444:1083-1087.

12. Senger, D.R., et al. 1983. Tumor cells secrete a vascular permeability factor that promotes accumulation of ascites fluid. Science. 219:983-985.

13. Eriksson, A., et al. 2002. Placenta growth factor1 antagonizes VEGF-induced angiogenesis and tumor growth by the formation of functionally inactive PIGF-1/VEGF heterodimers. Cancer Cell. 1:99-108.

14. Batchelor, T.T., et al. 2007. AZD2171, a pan-VEGF receptor tyrosine kinase inhibitor, normalizes tumor vasculature and alleviates edema in glioblastoma patients. Cancer Cell. 11:83-95.

15. Hurwitz, H., et al. 2004. Bevacizumab plus irinotecan, fluorouracil, and leucovorin for metastatic colorectal cancer. N. Engl. J. Med. 350:2335-2342.

16. Motzer, R.J., et al. 2007. Sunitinib versus interferon alfa in metastatic renal-cell carcinoma. N. Engl. J. Med. 356:115-124.

17. Sonpavde, G. 2003. Bevacizumab in renal-cell cancer [letter]. N. Engl. J. Med. 349:1674.

18. Cao, Y. 2005. Opinion: emerging mechanisms of tumour lymphangiogenesis and lymphatic metastasis. Nat. Rev. Cancer. 5:735-743.

19. Casanovas, O., Hicklin, D.J., Bergers, G., and Hanahan, D. 2005. Drug resistance by evasion of antiangiogenic targeting of VEGF signaling in late-stage pancreatic islet tumors. Cancer Cell. 8:299-309.

20. Hanahan, D., and Weinberg, R.A. 2000. The hallmarks of cancer. Cell. 100:57-70.

21. Westermark, B., and Heldin, C.H. 1993. Plateletderived growth factor. Structure, function and implications in normal and malignant cell growth. Acta Oncol. 32:101-105.

22. Nguyen, M., et al. 1994. Elevated levels of an angiogenic peptide, basic fibroblast growth factor, in the urine of patients with a wide spectrum of cancers. J. Natl. Cancer Inst. 86:356-361.

23. Lindahl, P., Johansson, B.R., Leveen, P., and Betsholtz, C. 1997. Pericyte loss and microaneurysm formation in PDGF-B-deficient mice. Science. 277:242-245.

24. Soriano, P. 1997. The PDGF alpha receptor is required for neural crest cell development and for normal patterning of the somites. Development. 124:2691-2700.

25. Shing, Y., et al. 1984. Heparin affinity: purification of a tumor-derived capillary endothelial cell growth factor. Science. 223:1296-1299.

26. Folkman, J., et al. 1991. Duodenal ulcer. Discovery of a new mechanism and development of angiogenic therapy that accelerates healing. Ann. Surg. 214:414-425; discussion 426-427.

27. Arbiser, J.L., et al. 2001. Differential expression of active mitogen-activated protein kinase in cutaneous endothelial neoplasms: implications for biologic behavior and response to therapy. J. Am. Acad. Dermatol. 44:193-197.

28. Cao, Y., and Cao, R. 1999. Angiogenesis inhibited by drinking tea. Nature. 398:381.

29. O'Reilly, M.S., et al. 1994. Angiostatin: a novel angiogenesis inhibitor that mediates the suppression of metastases by a Lewis lung carcinoma. Cell. 79:315-328.

30. Yoshida, A., Anand-Apte, B., and Zetter, B.R. 1996. Differential endothelial migration and proliferation to basic fibroblast growth factor and vascular endothelial growth factor. Growth Factors. 13:57-64.

31. Cao, R., et al. 2003. Angiogenic synergism, vascular stability and improvement of hind-limb ischemia by a combination of PDGF-BB and FGF-2. Nat. Med. 9:604-613.

32. Li, X., et al. 2005. Revascularization of ischemic tissues by PDGF-CC via effects on endothelial cells and their progenitors. J. Clin. Invest. 115:118-127. doi:10.1172/JCI200519189.

33. Cao, R., et al. 2002. Angiogenesis stimulated by PDGF-CC, a novel member in the PDGF family, involves activation of PDGFR-alphaalpha and alphabeta receptors. FASEB J. 16:1575-1583.

34. Millette, E., et al. 2005. Platelet-derived growth fac-
tor-BB-induced human smooth muscle cell proliferation depends on basic FGF release and FGFR-1 activation. Circ. Res. 96:172-179.

35. Kano, M.R., et al. 2005. VEGF-A and FGF-2 synergistically promote neoangiogenesis through enhancement of endogenous PDGF-B-PDGFRbeta signaling. J. Cell Sci. 118:3759-3768.

36. Sjoblom, T., et al. 2001. Growth inhibition of dermatofibrosarcoma protuberans tumors by the platelet-derived growth factor receptor antagonist STI571 through induction of apoptosis. Cancer Res. 61:5778-5783.

37. Schwartz, R. 2002. A molecular star in the wars against cancer. N. Engl. J. Med. 347:462-463.

38. Mancuso, M.R., et al. 2006. Rapid vascular regrowth in tumors after reversal of VEGF inhibition. J. Clin. Invest. 116:2610-2621. doi:10.1172/JCI24612.

39. Cao, R., et al. 1999. Suppression of angiogenesis and tumor growth by the inhibitor K1-5 generated by plasmin-mediated proteolysis. Proc. Natl. Acad. Sci.U. S. A. 96:5728-5733.

40. Roy, J., et al. 2002. Fibronectin promotes cell cycle entry in smooth muscle cells in primary culture. Exp. Cell Res. 273:169-177.

41. Afink, G., Westermark, U.K., Lammerts, E., and Nister, M. 2004. C/EBP is an essential component of PDGFRA transcription in MG-63 cells. Biochem. Biophys. Res. Commun. 315:313-318.

42. Ballagi, A.E., Ishizaki, A., Nehlin, J.O., and Funa, K. 1995. Isolation and characterization of the mouse PDGF beta-receptor promoter. Biochem. Biophys. Res. Commun. 210:165-173.

43. Molander, C., Hackzell, A., Ohta, M., Izumi, H., and Funa, K. 2001. Sp1 is a key regulator of the PDGF beta-receptor transcription. Mol. Biol. Rep. 28:223-233.

44. Arteaga, C.L., et al. 1991. Elevated content of the tyrosine kinase substrate phospholipase C-gamma 1 in primary human breast carcinomas. Proc. Natl. Acad. Sci. U. S. A. 88:10435-10439.

45. Cao, R., et al. 2004. PDGF-BB induces intratumoral lymphangiogenesis and promotes lymphatic metastasis. Cancer Cell. 6:333-345.

46. Soutter, A.D., Nguyen, M., Watanabe, H., and Folkman, J. 1993. Basic fibroblast growth factor secreted by an animal tumor is detectable in urine. Cancer Res. 53:5297-5299.

47. Bjorndahl, M.A., et al. 2005. Vascular endothelial growth factor-A promotes peritumoral lymphangiogenesis and lymphatic metastasis. Cancer Res. 65:9261-9268

48. Govindarajan, B., et al. 2005. Malignant transformation of human cells by constitutive expression of platelet-derived growth factor-BB. J. Biol. Chem. 280:13936-13943. 